\title{
Second trimester ultrasonographic diagnosis of ileal atresia, histopathological findings consistent with cystic fibrosis and negative genetic tests
}

\author{
Asher Bashiri, Avi Harlev, Eliezer Burstein \\ Esther Maor, Moshe Mazor, Reli Hershkowitz
}

\begin{abstract}
Introduction: Echogenic bowel is diagnosed in $0.2 \%$ to $1.4 \%$ of second trimester ultrasonographic examinations. This finding occurs as a normal variant in the second trimester but also has been associated with several pathologic conditions that include cystic fibrosis (CF), chromosomal abnormalities and in utero infection with cytomegalovirus and toxoplasmosis. The diagnosis of fetal echogenic bowel in second trimester fetuses has significant implications for prenatal management. It is strongly associated with adverse pregnancy outcome with utero-placental insufficiency, particularly in cases in which the maternal serum alphafetoprotein concentration is elevated due to severe feto-maternal bleeding. Case Report: A 30 year old Ashkenazi woman was admitted at 22 weeks of gestation due to recurrent vaginal bleeding. During prenatal care an elevated Elevated maternal serum AFP (MSAFP) as well
\end{abstract}

Asher Bashiri ${ }^{1}$, Avi Harlev ${ }^{1}$, Eliezer Burstein ${ }^{1}$, Esther Maor ${ }^{1}$, Moshe Mazor ${ }^{1}$, Reli Hershkowitz ${ }^{1}$

Affiliations: ${ }^{1}$ Department of Obstetrics and Gynecology, Soroka University Medical Center, and Faculty of Health Sciences Ben-Gurion University of the Negev, BeerSheva, Israel.

Corresponding Author: Asher Bashiri, Department of Obstetrics and Gynecology, Soroka University Medical Center, P.O Box 151, Beer-Sheva 64101, Israel; Phone: +972-8-640-5151; Fax: +972-8-640-3294; E-mail: abashiri@bgu.ac.il

Received: 13 October 2010

Accepted: 5 November 2010

Published: 19 December 2010 as hyperechogenic bowel were observed. Ultrasound at her admission showed fetal distended bowel with a lower abdominal mass appearance with hyperechogenic foci. Amniocentesis was performed, with normal fetal karyotype; Cytomegalovirus and cystic fibrosis (CF) testing. The mother was positive for $\mathrm{CF}$, but both the husband and the fetus were negative. At the 24th week, she delivered a male fetus with abdominal distention. X-ray and abdominal ultrasound showed obstruction at the level of the terminal ileum. The newborn died 18 hours after delivery. A postmortem examination revealed no evidence of external malformations and atresia of the terminal ileum with total obstruction of the intestinal lumen; near the atretic portion changes consistent with meconium ileus were seen. Foci of mucous deposition on the intestinal surface and calcifications were observed, all features highly suggestive of CF. Our presentation discusses the prenatal ultrasonographic findings in non-duodenal bowel obstruction and histopathological findings in CF. This case is unique in the early detection of fetal bowel obstruction by ultrasound and the discrepancy between the histological and genetic results for CF. Conclusion: Echogenic bowel is associated with several serious conditions including $\mathrm{CF}$ that should be ruled out once the diagnosis of echogenic bowel is made, although it is subjective. These include fetal karyotype, infections, structural malformations, thalasemia, CF and placental damage. CF etiology is very important for genetic consultations for the next pregnancies and it should be diagnosed by examining the known mutations. However, a pathological examination of available bowel tissue can highly support the diagnosis. 
Keywords: Echogenic bowel, Cystic fibrosis, Ileal atresia, Alpha-fetoprotein

$* * * * * * * * *$

Bashiri A, Harlev A, Burstein E, Maor E, Mazor M, Hershkowitz R. Second trimester ultrasonographic diagnosis of ileal atresia, histopathological findings consistent with cystic fibrosis and negative genetic tests. International Journal of Case Reports and Images 2010;1(4):12-15.

$* * * * * * * * *$.

doi:10.5348/ijcri-2010-12-11-CR-3

\section{INTRODUCTION}

Echogenic bowel is diagnosed in $0.2 \%$ to $1.4 \%$ of second trimester ultrasonographic examinations. This finding occurs as a normal variant in the second trimester but also has been associated with several pathologic conditions that include cystic fibrosis (CF), chromosomal abnormalities, in utero infection with cytomegalovirus, toxoplasmosis and other viruses, thalasemia and structural malformations, especially bowel malformations [1,2]. Fetal echogenic bowel should be considered also as an important marker of placental damage. This finding in the second trimester is highly associated with adverse pregnancy outcome with utero-placental insufficiency, particularly in cases in which the MSAFP concentration is elevated due to severe feto-maternal bleeding [3]. Ultrasound assessment of echogenic bowel is subjective usually comparing the echogenicity with adjacent bone, or liver [1]. The diagnosis of fetal echogenic bowel in second trimester fetuses has significant implications for prenatal management.

The purpose of this case report is to describe the ultrasonographic early detection of fetal bowel obstruction and the discrepancy between histological and genetic results for $\mathrm{CF}$.

\section{CASE REPORT}

A 30 year old multipara of Ashkenazi ascendance woman was admitted to our obstetric department at 22 $4 / 7$ weeks of gestation due to recurrent vaginal bleeding. Her past gynecological and obstetric history included regular menses, six pregnancies, three of which ended by induced abortions, one spontaneous abortion and a vaginal delivery of a normal fetus at term. Prenatal care included a first ultrasound at 17 weeks, a triple test done at 19 weeks of pregnancy with an elevated MSAFP (2.88 MOM) with normal HCG and UE3. Amniocentesis was recommended but the patient declined. A second trimester fetal anatomy scan at $202 / 7$ weeks of pregnancy revealed a hyperechogenic bowel.

At 22 4/7 weeks of gestation she was seen by an obstetrician due to mild vaginal bleeding which recurred the same day. She presented to the emergency room where she was admitted for observation.

At admission the physical examination was normal except for mild vaginal bleeding. A Pap smear was taken which later returned normal. The tocograph showed uterine contractions and the ultrasound performed at admission revealed a cervical length of 35 $\mathrm{mm}$.

Blood samples were drawn (CBC, PT/PTT and fibrinogen levels) and the patient received intravenous fluids. The contractions and vaginal bleeding ceased and blood samples returned within the normal range. She was sent to the ward for observation.

During the second admission day an ultrasonographic evaluation was performed at the ultrasound department which showed an anterior placenta with no signs of abruption.

Fetal biometry demonstrated a two week growth lag, fetal distended bowel with a lower abdominal mass appearance with hyperechogenic foci within, no ascites or calcifications, normal amniotic fluid and no other fetal anatomic abnormalities (Figure 1). The same day a fetal echocardiogram was performed which was normal.

Due to the findings the couple underwent genetic counseling including $\mathrm{CF}$. The mother was found to be positive for the mutation: $\Delta \mathrm{F}-508$.

Genetic amniocentesis and tests to rule out STORCH were performed. Another ultrasonographic evaluation was done the third day of after admission which revealed the same findings, with no ascites or calcifications and a normal amount of amniotic fluid.

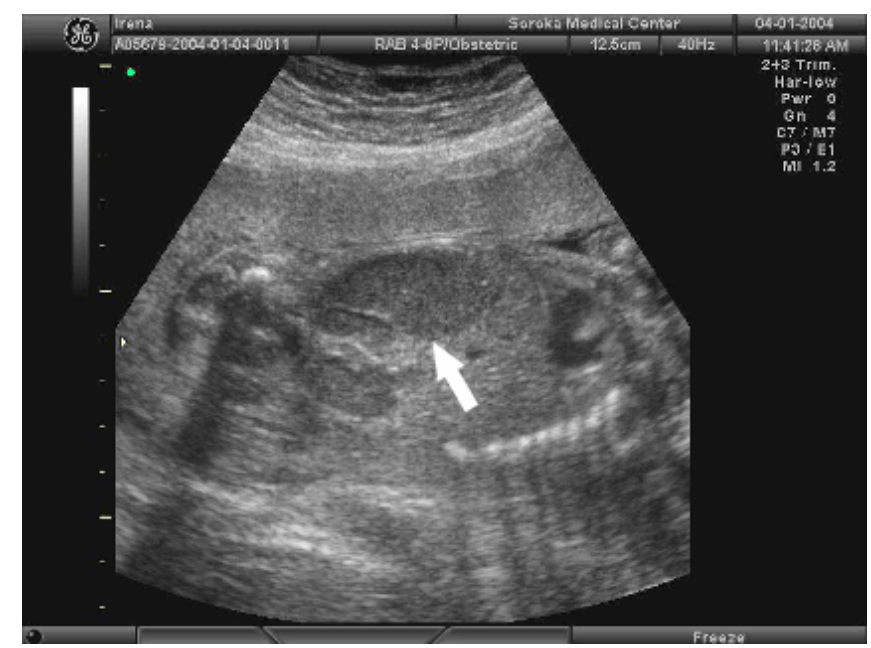

Figure 1. Sonographic exam showing the intra-abdominal mass with echogenic foci. 


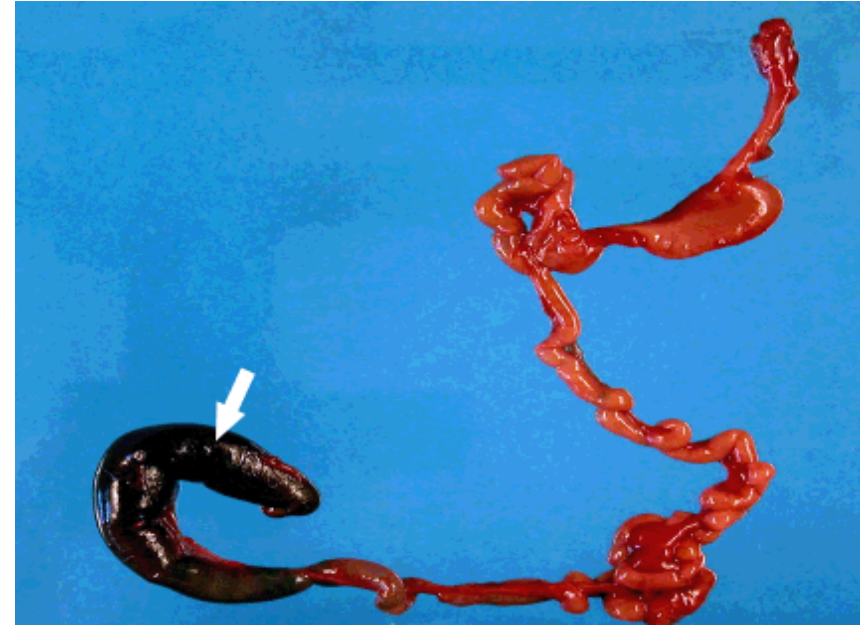

Figure 2. Atresia of the terminal ileum with total obstruction of the intestinal lumen.

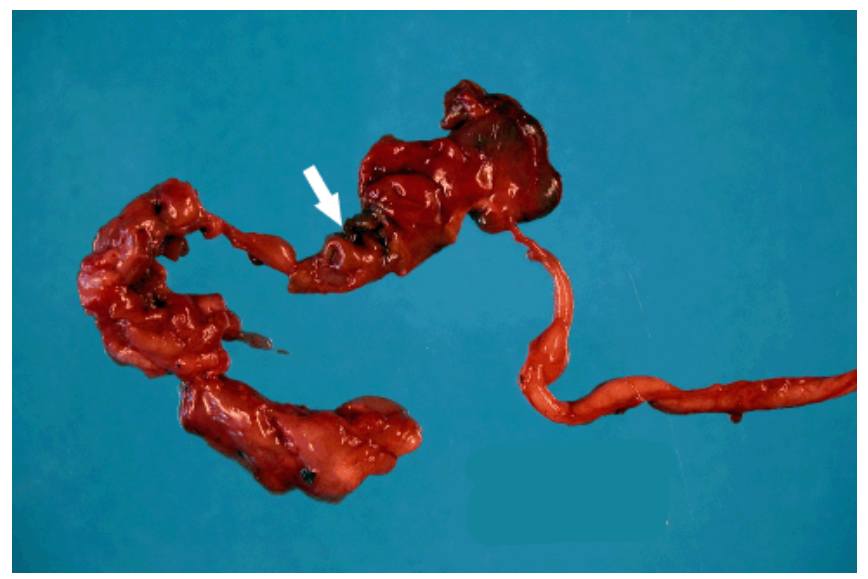

Figure 3. Perforation of the ileum (arrow).

Maternal test for Toxoplasmosis was negative (ELISA), IgM for CMV was negative and IgG positive. IgG for

Herpes simplex 1 was positive. Amniotic fluid tests for CMV (Shell-Vial and PCR) returned negative. During her hospital stay vaginal bleeding continued with no alteration in subsequent coagulation tests.

At the $24^{\text {th }}$ week ( $11^{\text {th }}$ day after admission) premature rupture of membranes ensued and 4 hours later she was transferred to the delivery room with regular contractions, the cervix fully effaced and $8 \mathrm{~cm}$ of dilation. Fifty minutes later she delivered a male fetus weighing 572 grams with an Apgar score of 6 at the first minute and 7 at the fifth minute. Resuscitation was needed immediately after delivery; the newborn was intubated and transferred to the neonatal intensive care unit (NICU).

The newborns physical examination revealed abdominal distention and severe signs of prematurity consistent with 23-24 gestational weeks. In the NICU the newborn received therapy with surfactant $100 \%$ oxygen with partial response.

The abdominal distension worsened and abdominal $\mathrm{X}$-ray showed intestines without air, after adding contrast the abdominal cavity and the proximal small bowel were identified with suspicion of obstruction at the level of the terminal ileus. An abdominal ultrasound showed severely distended bowel with a liquid content and without calcifications. The newborn died 18 hours after delivery. The maternal post-partum period was normal and the patient was discharged after 48 hrs.

A postmortem examination of the fetus revealed no evidence of external malformations and atresia of the terminal ileum with total obstruction of the intestinal lumen; near the atretic portion changes consistent with meconium ileus were seen. Foci of mucous deposition on the intestinal surface and calcifications were observed, all features highly suggestive of CF (Figures $2,3)$.

\section{DISCUSSION}

The fetal bowel when screened in the longitudinal and transverse plain shows echogenicity that is similar to or greater than the surrounding bone. This feature normally presents between 16-20 weeks of gestation but usually resolves after this period. The true echogenicity of fetal tissues is hard to ascertain in utero making the correct assessment of fetal bowel echogenicity difficult even in experienced hands. The diagnosis of this condition therefore is highly subjective and likely to be associated with substantial inter-and intra-observer variability [1]. Increased echogenicity of the fetal bowel has been described in association with several conditions including, aneuploidy, infections, growth restriction, cystic fibrosis [4], bowel malformations [1] and intraamniotic bleeding (5). Sepulveda et al. reported 34\% of poor perinatal outcome in fetuses having Echogenic bowel [2].

Ewer et. al. [6] speculated that echogenic bowel was a reflection of intrauterine gut ischemia because gut dysfunction was noted in fetuses with echogenic bowel. Other mechanisms underlying fetal hyperechogenic bowel are bowel hypotonia with excessive desiccation of meconium as in Down syndrome, viscid meconium as in intrauterine growth restriction or cystic fibrosis, hypertrophied bowel and external compression by a tumor or ascites. Therefore, when considering differential diagnosis for fetal echogenic bowel it is necessary to scan for other markers of aneuploidy, assessment of growth, screen for infections and assess the risk status for cystic fibrosis as well as structural malformations, especially gastrointestinal $[1,7,8]$. Moreover, Yuval et. al. [8] reported fifteen cases of echogenic fetal bowel associated with maternal vaginal bleeding. It is not clear however, why fetal echogenic bowel is found in association with vaginal bleeding. At 
least one possible explanation is the access of blood or blood products to the amniotic fluid. The echogenicity in these cases is most likely secondary to ingested blood in the fetal gastrointestinal tract.

Achiron et. al. [3] reported that maternal serum alpha fetoprotein was significantly higher in pregnancies complicated by fetal echogenic bowel suggesting placental dysfunction. This group of patients has also been reported to have a poor outcome. In addition, featl echogenic bowel was found to be the only finding in only $5 \%$ of the fetuses with an adverse outcome [9].

Our case presents fetal echogenic bowel which can be related to some of the conditions mentioned as causes of this sonographic finding. First, the vaginal bleeding and the two week lag between gestational age and fetal biometry, on the other hand the mother was a known carrier of the CF mutation and although the fetus screened negative for the common CF mutations of our population, histological findings were consistent with CF. The etiology for the pathologies in our case are not certain due to the mixed findings including vaginal bleeding, an elevated MSAFP levels, intrauterine growth restriction, bowel malformation and negative results for $\mathrm{CF}$ mutations in the genetic screening but pathological findings consistent with CF. There is a possibility that the fetus was a carrier of an uncommon $\mathrm{CF}$ gene mutation in our population which was not identified or a mutation not yet identified.

\section{CONCLUSION}

Echogenic bowel is associated with several serious conditions including CF that should be ruled out once the diagnosis of echogenic bowel is made, although it is subjective. These include fetal karyotype, infections, structural malformations, thalasemia, CF and placental damage. CF etiology is very important for genetic consultations for the next pregnancies and it should be diagnosed by examining the known mutations. Moreover, a pathological examination of available bowel tissue can highly support the diagnosis.

$$
* * * * * * * * *
$$

\section{Author Contributions}

Asher Bashiri - Conception and design, Acquisition of data, Analysis and interpretation of data, Drafting the article, Critical revision of the article, Final approval of the version to be published

Avi Harlev - Conception and design, Drafting the article, Critical revision of the article, Final approval of the version to be published

Eliezer Burstein - Conception and design, Drafting the article, Critical revision of the article, Final approval of the version to be published Esther Maor - Conception and design, Acquisition of data, Analysis and interpretation of data, Critical revision of the article, Final approval of the version to be published

Moshe Mazor - Conception and design, Acquisition of data, Analysis and interpretation of data, Critical revision of the article, Final approval of the version to be published

Reli Hershkowitz - Conception and design, Acquisition of data, Analysis and interpretation of data, Drafting the article, Critical revision of the article, Final approval of the version to be published

\section{Guarantor}

The corresponding author is the guarantor of submission.

\section{Conflict of Interest}

Authors declare no conflict of interest.

\section{Copyright}

(C) Asher Bashiri et. al. 2010; This article is distributed under the terms of Creative Commons attribution 3.0 License which permits unrestricted use, distribution and reproduction in any means provided the original authors and original publisher are properly credited. (Please see www.ijcasereportsandimages.com /copyright-policy.php for more information.)

\section{REFERENCES}

1. Bashiri A, Burstein E, Hershkowitz R, Mazor M.Fetal echogenic bowel by ultrasound: what is the clinical significance? Harefuah. 2007; 146:964-9, 996-997.

2. Sepuvelda W, Sebire N.J. Fetal Echogenic bowel: a complex scenario. Ultrasound obstet gynecol 2000;16:510-514.

3. Achiron R, Seidman DS, Horowitz A, Mashiach S, Goldman B, Lipitz S. Hyperechogenic fetal bowel and elevated serum alphafetoprotein: a poor fetal prognosis. Obstet Gynecol 1996;88:368-371.

4. Irish M, Ragi JM, Karamanoukian H, Borowitz DS, Scmidt D, Glick PL. Prenatal diagnosis of the fetus with cystic fibrosis and meconium ileus. Pediatr Surg Int 1997;12:434-436.

5. Petrikovsky B, Smith-Levitin M, Holsten N. Intraamniotic bleeding and fetal echogenic bowel. Obstet Gynecol 1999;93:684-686.

6. Ewer Ak, McHugo J, Chapman S, Newwel SJ. Fetal echoenic gut: A marker of intrauterine gut ischemia? Arch Dis Child 1993;69:510-513.

7. Bashiri A, Burstein E, Hershkowitz R, Maor E, Landau D, Mazor M..Fetal echogenic bowel at 17 weeks' gestational age as the early and only sign of a very long segment of Hirschsprung disease. J Ultrasound Med 2008;27:1125-1126.

8. Yaron Y, Hassan S, Geva E, Kupferminc MJ, Yavetz $\mathrm{H}$, Evans MI. Evaluation of fetal echogenic bowel in the second trimester. Fetal Diagn Ther 1999;14:176180.

9. Iruretagoyena JI, Bankowsky $\mathrm{H}$, Heiser $\mathrm{T}$, Birkeland L, Grady M, Shah D. Outcomes for fetal echogenic bowel during the second trimester ultrasound.J Matern Fetal Neonatal Med 2010;23:1271-1273. 